\title{
Vascular Lactic Acid Infusions Do Not Alter the Incidence of Fetal Breathing Movements or Their Inhibition by Acute Hypoxemia
}

\author{
A. ROGER HOHIMER AND JOHN M. BISSONNETTE
}

Department of Obstetrics and Gynecology, Oregon Health Sciences University, Portland, Oregon 97201

\begin{abstract}
Hypoxemia transiently inhibits the incidence of fetal breathing movements (FBM), but their incidence returns to normal after several hours despite maintained hypoxemia. We hypothesized that the lactic acidosis associated with prolonged systemic hypoxemia might mediate the adaptation of the hypoxemic inhibition of FBM. In sheep fetuses, the incidence of FBM was measured in a control hour and during $6 \mathrm{~h}$ of i.v. infusion of $\mathrm{t}$-lactic acid, which raised the blood lactate concentrations to levels seen with moderate hypoxemia. FBM were observed at the same incidence as during control during each of the first $4 \mathrm{~h}$ (all approximately $40 \%$ ). In the 5 th $h$ of lactic acid infusion, fetal hypoxemia was induced by lowering maternal inspired oxygen fraction and FBM occurred only $8 \pm 1 \%$ (SEM) of that hour. In a subsequent normoxemic recovery hour, the incidence of FBM remained below control levels. In the same animals on a different day, a similar hypoxemia induced without the acid infusion caused a comparable inhibition of FBM, but the incidence of FBM returned to the control level in a subsequent recovery hour. A moderate peripheral lactic acidosis does not blunt the inhibition of FBM evoked by acute hypoxemia and is not a likely explanation for the return of FBM during prolonged hypoxemia but actually might mediate some of the inhibition. (Pediatr Res 29: 483-486, 1991)
\end{abstract}

\section{Abbreviations}

FBM, fetal breathing movements $L V$, low voltage

In unanesthetized fetal sheep, acute hypoxia causes a depression in the incidence of FBM (1-4). However, with several different kinds of induced, prolonged hypoxemia, FBM do not remain inhibited but return toward control incidence after several hours (5-7). We have suggested that the peripheral lactic acidosis that accompanies prolonged hypoxemia might override the initial inhibition of FBM that occurs with acute hypoxemia (6).

We have sought to determine if a continuous lactic acid infusion could alter the inhibition of FBM associated with acute hypoxemia. Such a result might implicate the lactic acidosis that can be generated endogenously during hypoxemia with the return of FBM seen in prolonged hypoxemia.

Received August 8, 1990; accepted December 12, 1990.

Correspondence: A. Roger Hohimer, Ph.D., Rm. 428, Research Bldg., Mail Stop

L-458, Oregon Health Sciences University, Portland, OR 97201.

Supported by National Institute of Health Grant HL-38039.

\section{MATERIALS AND METHODS}

Surgery. Seven pregnant ewes were used for these studies. All procedures and experiments were reviewed and approved by the Institutional Animal Care and Use Committee. Sterile surgery was carried out between 121 and $126 \mathrm{~d}$ gestational age, under nitrous oxide and fluothane anesthesia in oxygen. The methods used for placement of catheters in the fetal trachea, axillary artery, sagittal vein, hind limb vein, and amniotic fluid, and placement of electrodes above the dura have been described in detail (6). A polyvinyl catheter was inserted in the ewe's trachea and sutured to the s.c. tissues. After surgery, the animals were housed in $1.5 \times 3 \mathrm{~m}$ pens with free access to food and water.

Physiologic measurements. Fetal tracheal, amniotic fluid, and arterial catheters were connected to pressure transducers (model $23 \mathrm{Db}$; Statham, Oxnard, CA) that were calibrated with a mercury manometer. All pressures were recorded on a strip chart recorder. The fetal EEG was amplified and filtered (highpass 0.5 $\mathrm{Hz}$ ) and displayed on the recorder.

Blood analysis. Fetal blood samples were analyzed for $\mathrm{pH}, \mathrm{PO}_{2}$ and $\mathrm{PCO}_{2}$ at $39^{\circ} \mathrm{C}$ with standard electrodes (Radiometer Ltd., Copenhagen, Denmark). Blood glucose and lactate concentrations were determined by glucose and lactate oxidase methods (YSI model 23; Yellow Springs Instruments, Yellow Springs, $\mathrm{OH})$ as previously described (6).

Protocol. Experiments were initiated 4-8 d after surgery and carried out over the following 2-6 d. The fetuses were between 127 and $142 \mathrm{~d}$ gestation at the time experiments were performed. The studies were conducted between 1000 and $1400 \mathrm{~h}$, a period during which we have not observed differences in the incidence of fetal breathing (8). The animals ate ad libitum during the studies.

Two studies were performed on each fetus on different days in a mixed order. In one protocol, after a 1 -h control period, fetal lactic acidemia was induced by infusion of $3.2 \mathrm{M}$ L-lactic acid (Sigma Chemical Co., St. Louis, MO) at $13.3 \mathrm{~mL} / \mathrm{h}$ into the venous catheter for $6 \mathrm{~h}$. To induce fetal hypoxemia in the 5 th $\mathrm{h}$ of lactic acidosis, nitrogen and carbon dioxide were added the ewe's inspired air by perfusing the maternal trachea (9). The flow rates were $8-11 \mathrm{~L} / \mathrm{min}$ for nitrogen and $0.8-0.9 \mathrm{~L} / \mathrm{min}$ for $\mathrm{CO}_{2}$. In the final (6th) hour of lactic acid infusion, the ewe's inspired gas was returned to normal. This is referred to as a "recovery" hour. Air was not infused during the control, infusion, or recovery periods. Fetal arterial blood samples were taken in the control hour, after 1 and $4 \mathrm{~h}$ of infusion, and at the midpoint of the hypoxemic hour and the normoxemic recovery hour.

In the other protocol, FBM and EEG were recorded during a control hour, a hypoxemic hour, and a normoxemic recovery hour. Arterial blood samples were taken halfway through each hour.

Analysis. FBM were defined as repeated transient negative pressures recorded in the trachea (relative to amniotic fluid) that were occurring at least $0.25 \mathrm{~Hz}$ and at least $0.13 \mathrm{kPa}$ in amplitude 
or depth. Breathing depth was measured as the amplitude of the tracheal peak negative pressure deflection measured off the strip chart. Breaths were selected at 12-s intervals when FBM occurred and averaged to yield a single value for breathing depth for each 6 -min period of breathing. Ten of these interim average depths were averaged together to form a composite hourly depth for each animal. The number of breaths collectively used to generate this mean varied according to the incidence of FBM but ranged from 14 to 210 breaths for each hour. The estimates of variation reported reflect the interanimal distribution. The incidence of FBM was determined by dividing the time when FBM was present by the 60 -min observation time. Fetal EEG was assessed visually into periods of $\mathrm{LV}(<50 \mu \mathrm{V})$ and high voltage $(50-200$ $\mu \mathrm{V})$. The incidence of LV EEG was similarly calculated. The incidence of FBM in LV EEG was calculated by dividing the time FBM were observed in LV EEG by the total time in that EEG pattern in the hour of observation. The EEG was analyzed in only five of the seven animals because in the other two highfrequency noise and baseline drift were present due to a malfunctioning amplifier. Hourly mean blood pressure and heart rate were determined by the average of values selected at 2-min intervals over each hour.

All data are reported as the means \pm SEM. Inference testing was performed using one-way analysis of variance and significant differences from control values were evaluated using the protected Least Significant Difference test (10).

\section{RESULTS}

Table 1 reports the arterial blood gas tensions, $\mathrm{pH}$, and lactate and glucose concentrations during control and after 1 and $4 \mathrm{~h}$ of lactic acid infusion, during the last $30 \mathrm{~min}$ of hypoxemia, and after $30 \mathrm{~min}$ of recovery. Glucose levels rose in conjunction with the lactate infusion. Fetal heart rate tended to increase with the acid infusion, but this did not reach statistical significance until the recovery hour. Mean arterial blood pressure also tended to increase, but the increase was not statistically significant.

Figure 1 shows the incidence of FBM (panel $A$ ), the LV EEG state (panel $B$ ), and FBM during the LV state (panel $C$ ). Data is grouped for a 1-h control period and then $6 \mathrm{~h}$ of lactic acid infusion. There was an increase in the incidence of FBM in the first hour, but no difference from control was seen in the incidence of FBM for the next $3 \mathrm{~h}$ of lactic acid infusion. The increase in the percent of the LV EEG state having FBM paralleled the incidence of FBM, inasmuch as there was no difference in the incidence of LV EEG. The 1-h period of induced hypoxemia inhibited FBM, and this inhibition persisted in a subsequent recovery hour. Hypoxemia did not alter the incidence of the LV EEG. Because virtually no FBM occurred in the high voltage EEG in any instance, the effect of hypoxemia was on the incidence of FBM in the LV EEG pattern

The depth of FBM during the control hour was $0.76 \pm 0.03$
$\mathrm{kPa}$. Lactic acid infusion alone caused a slight but significant increase in the depth in the first hour $(0.97 \pm 0.05 \mathrm{kPa})$, but the breath amplitudes for the next $3 \mathrm{~h}$ were not different from control $(0.75 \pm 0.07,0.80 \pm 0.04$, and $0.73 \pm 0.06 \mathrm{kPa}$ respectively). Hypoxemia reduced the amplitude of FBM to $0.59 \pm 0.05 \mathrm{kPa}$. The depth of breathing in the recovery hour was not different from control $(0.66 \pm 0.08 \mathrm{kPa})$.

Figure 2 illustrates the incidence of FBM (panel $A$ ), the LV EEG (panel $B$ ) and FBM during LV EEG (panel $C$ ) in these same fetuses (on a different day). The data is reported from a 1$\mathrm{h}$ control period, a 1-h period of induced hypoxemia, and a normoxic recovery hour. Hypoxemia was associated with a reduced incidence of FBM with no change in the incidence of the LV EEG pattern, leading to a dramatic fall in the incidence of FBM in the LV EEG pattern. All of these changes were comparable to those seen in the lactic acid infusion protocol (Fig. 1). In contrast, the incidence of FBM in the posthypoxic recovery hour returned to a percentage not distinguishable from control.

In the hypoxemic (no acid infusion) protocol, the depth of FBM in the control hour was not different $(0.85 \pm 0.07 \mathrm{kPa})$ than in the lactic acid protocol control period. Hypoxemia significantly $(p \leq 0.05)$ reduced the amplitude to $0.59 \pm 0.04$ $\mathrm{kPa}$, whereas the recovery hour returned to a level not distinguishable from control.

Table 2 reports the average blood gas, $\mathrm{pH}$, lactate, and glucose concentrations, and heart rate and blood pressure values recorded during this protocol. The control gases and $\mathrm{pH}$ were no different from those reported in Table 1. During hypoxemia, there was a fall in arterial $\mathrm{PO}_{2}$ comparable to that seen during the hypoxemic hour of the lactic acid infusion protocol.

\section{DISCUSSION}

Hypoxic ventilatory depression has recently been reviewed and several nonexclusive mechanisms proposed (11). Hypoxia may inhibit ventilation by acting either at higher or brainstem respiratory centers directly or by eliciting increased levels of some neuromodulatory chemical ( $\gamma$-aminobutyric acid or adenosine). Alternatively, hypoxia may alter the brain metabolism, blood flow, or bicarbonate concentrations to reduce the central chemical stimulation of either $\mathrm{CO}_{2}$ or hydrogen ion. Similarly, the escape from hypoxic inhibition of FBM may be due to the adaptation of some inhibitory neuromodulating element or by a progressive return of hydrogen ion stimulus to central chemoreceptors. The role of lactic acid is particularly difficult to evaluate because it could act either as a stimulator (hydrogen ion) or as an inhibitory neuromodulator (12). Additionally, systemic and brain lactic acidosis are a somewhat delayed sequel of hypoxemia and may persist for some time after a return to normoxemia.

The inhibition of FBM seen with acute anemic hypoxia and uterine asphyxia was transient despite the maintenance of lowered blood oxygen contents $(5,6)$. Similar returns of fetal respi-

Table 1. Arterial blood gases, $p H$, glucose, lactate before and during lactate infusion, and hypoxemia*

\begin{tabular}{|c|c|c|c|c|c|c|c|}
\hline & \multirow[b]{2}{*}{ Control } & \multicolumn{6}{|c|}{ Lactic acid infusion } \\
\hline & & $1 s t h$ & 2nd h & $3 \mathrm{rd} \mathrm{h}$ & 4 th $\mathrm{h}$ & $\begin{array}{l}\text { Hypoxemia } \\
(5 \text { th } \mathrm{h})\end{array}$ & $\begin{array}{l}\text { Recovery } \\
(6 \text { th } \mathrm{h})\end{array}$ \\
\hline $\mathrm{pHa}$ & $7.37 \pm 0.01$ & $7.28 \pm 0.01 \dagger$ & & & $7.23 \pm 0.02 \dagger$ & $7.20 \pm 0.02 \dagger$ & $7.15 \pm 0.02 \dagger$ \\
\hline $\mathrm{PaO}_{2}(\mathrm{kPa})$ & $2.5 \pm 0.1$ & $2.9 \pm 0.1$ & & & $2.7 \pm 0.1$ & $2.0 \pm 0.2 \dagger$ & $2.7 \pm 0.2$ \\
\hline $\mathrm{PaCO}_{2}(\mathrm{kPa})$ & $6.6 \pm 0.1$ & $6.9 \pm 0.1$ & & & $7.0 \pm 0.1$ & $6.6 \pm 0.2$ & $6.7 \pm 0.1$ \\
\hline Lactate (mM) & $1.5 \pm 0.1$ & $5.5 \pm 0.3 \dagger$ & & & $8.8 \pm 0.8 \dagger$ & $11.0 \pm 0.8 \dagger$ & $13.0 \pm 1.0 \dagger$ \\
\hline Glucose (mM) & $1.1 \pm 0.1$ & $1.4 \pm 0.1 \dagger$ & & & $1.4 \pm 0.1 \dagger$ & $1.5 \pm 0.1 \dagger$ & $1.5 \pm 0.1 \dagger$ \\
\hline $\begin{array}{l}\text { Mean blood pressure } \\
(\mathrm{kPa})\end{array}$ & $6.0 \pm 0.3$ & $6.3 \pm 0.3$ & $6.5 \pm 0.3$ & $6.5 \pm 0.3$ & $6.5 \pm 0.1$ & $6.7 \pm 0.1$ & $6.6 \pm 0.1$ \\
\hline $\begin{array}{l}\text { Heart rate (beats/ } \\
\text { min) }\end{array}$ & $179 \pm 6$ & $191 \pm 7$ & $191 \pm 6$ & $183 \pm 5$ & $181 \pm 4$ & $158 \pm 8$ & $195 \pm 8 \dagger$ \\
\hline
\end{tabular}

* Data are mean $\pm \mathrm{SEM}(n=7) . \mathrm{PaO}_{2}$, arterial $\mathrm{O}_{2}$ tension; $\mathrm{PaCO}_{2}$ arterial $\mathrm{CO}_{2}$ tension.

$\dagger$ Indicates different from control at the $p<0.05$ level. $(0.13 \mathrm{kPa} / \mathrm{mm} \mathrm{Hg})$. 

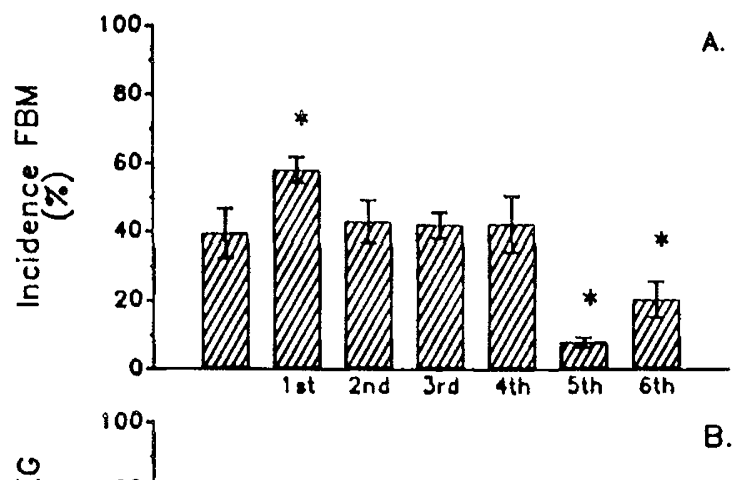

B.
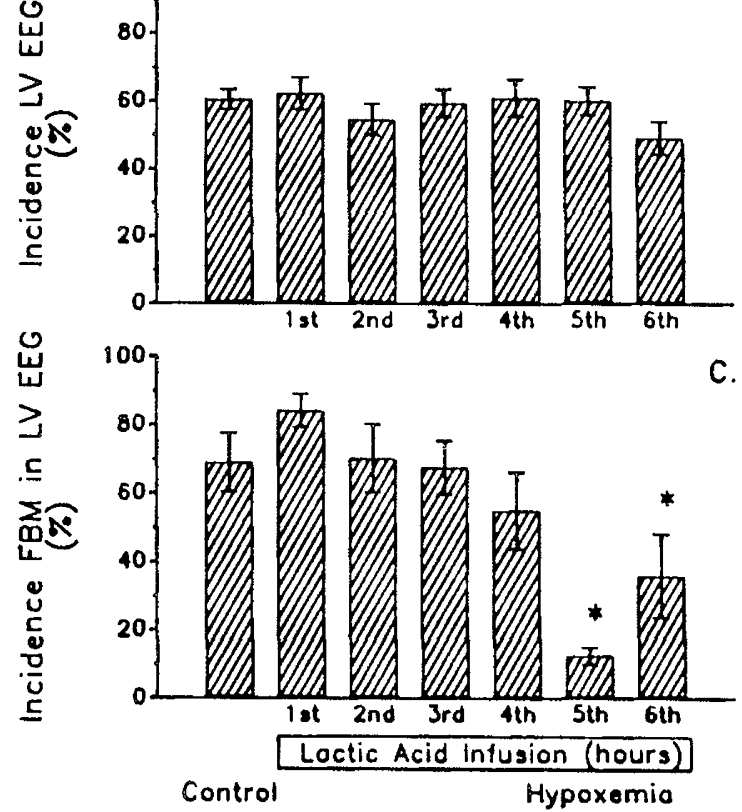

Fig. 1. Incidence of FBM, LV, EEG, and FBM during LV EEG. Data are for $1 \mathrm{~h}$ before (control) and $6 \mathrm{~h}$ of lactic acid infusion. In the 5 th $\mathrm{h}$, hypoxemia was induced. Bars represent means \pm 1 SEM. $n=7$ for panel $A$ and 5 for panels $B$ and $C$. ${ }^{*}$, difference from control, $p<0.05$.

ratory activity during hypoxemia have been reported by Koos et al. (7), although the time course of the return was longer. We have speculated that one possible mechanism of this return is the secondary metabolic acidosis of the blood and eventually the brain that results from the developing lactic acidosis.

Such a process has precedent or analogy with regard to the ventilatory acclimatization to prolonged hypoxemia in adult humans and animals (13), whereby initial ventilatory stimulation is followed by progressive, secondary (in time) increases in ventilation. The mechanism of this delayed stimulation of "adaptation" (14) remains controversial. It might be due to a central acidosis or neuromodulation, but there also is evidence that the carotid bodies are important to this ventilatory acclimatization, at least in goats (15).

Based on previous results by ourselves (8) and others (16), the 5 th $h$ of acid infusion in the absence of hypoxemia would be expected to stimulate $\mathrm{FBM}$. With $\mathrm{HCl}$ infusions, the incidence of FBM was elevated after $4 \mathrm{~h}$ of metabolic acidosis (8), a result similar to that reported by Molteni et al. (16) with $\mathrm{NH}_{4} \mathrm{Cl}$ infusions. The delay in the stimulation might have been due to a time delay before the blood acidosis manifested an acidosis at zentral chemoreceptors (17). In this current study, the lack of stimulation of FBM in the hours before and after hypoxemia argue against any role for lactic acid in mediating the adaptation jeen in previous hypoxemic experiments (5-7). However, some zaution should be exercised in interpreting these results. Evidence or a unique stimulatory effect of $\mathrm{HCl}$ as compared with other ixed acids also exists in studies of adult animals (18). There
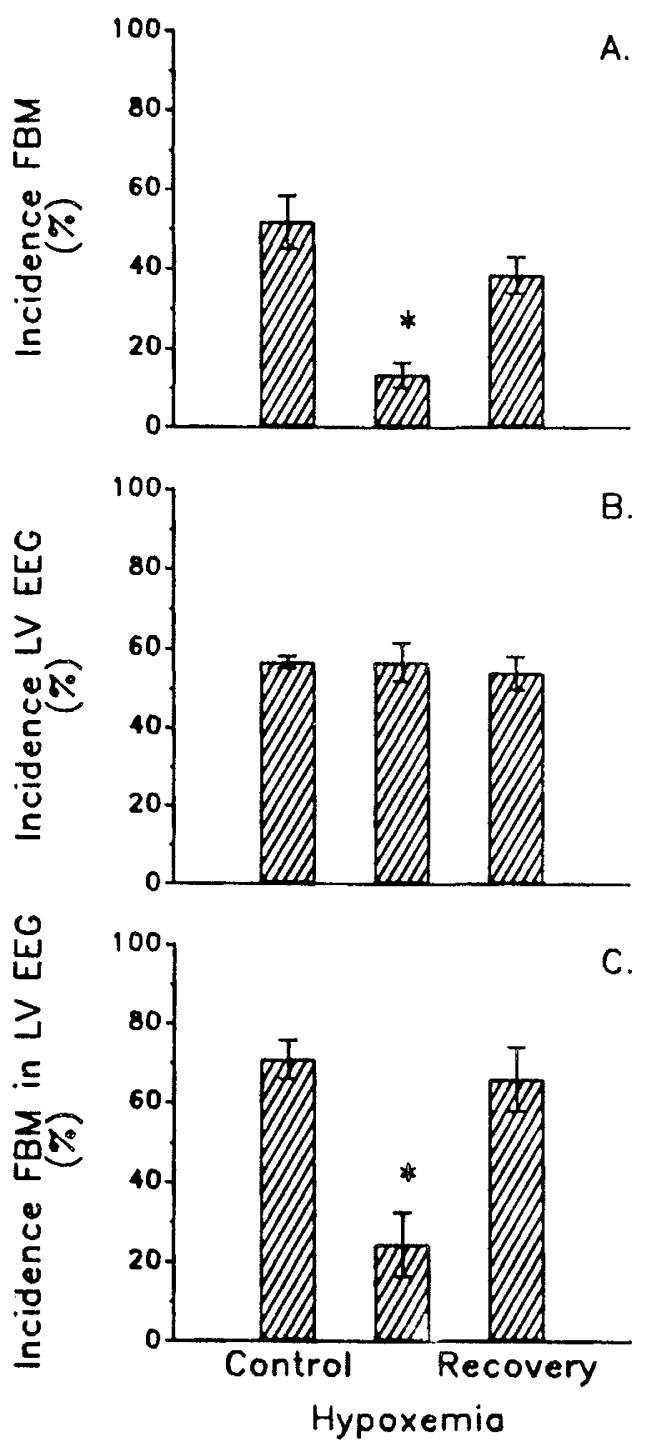

Fig. 2. Incidence of FBM (panel $A$ ), LV EEG (panel $B$ ), and FBM during LV EEG (panel $C$ ). Data are for $1 \mathrm{~h}$ before (Control), during (Hypoxemia), and after (Recovery) a $1-\mathrm{h}$ induced hypoxemia. Bars represent means \pm 1 SEM. $n=7$ for panel $A$ and 5 for panels $B$ and $C$. $*$, difference from control, $p<0.05$.

Table 2. Arterial blood gases, $p H$, and glucose, and lactate before, during, and after induced hypoxemia*

\begin{tabular}{lccc}
\hline & Control & Hypoxemia & Recovery \\
\hline $\mathrm{pH}$ & $7.38 \pm 0.01$ & $7.38 \pm 0.01$ & $7.37 \pm 0.01$ \\
$\mathrm{PaO}_{2}(\mathrm{kPa})$ & $2.6 \pm 0.1$ & $1.8 \pm 0.1 \dagger$ & $2.7 \pm 0.1$ \\
$\mathrm{PaCO}_{2}(\mathrm{kPa})$ & $6.4 \pm 0.2$ & $5.9 \pm 0.2$ & $6.3 \pm 0.1$ \\
$\mathrm{Lactate}(\mathrm{mM})$ & $1.4 \pm 0.1$ & $2.5 \pm 0.2 \dagger$ & $2.1 \pm 0.2 \dagger$ \\
Glucose $(\mathrm{mM})$ & $1.3 \pm 0.1$ & $1.3 \pm 0.1$ & $1.2 \pm 0.1$ \\
Mean blood pressure & $6.1 \pm 0.3$ & $6.0 \pm 0.3$ & $6.1 \pm 0.4$ \\
$\quad(\mathrm{kPa})$ & & & \\
Heart rate (beats/ & $174 \pm 5$ & $176 \pm 4$ & $190 \pm 4 \dagger$ \\
$\quad$ min) & & & \\
\hline
\end{tabular}

* Data are mean $\pm \mathrm{SEM}(n=7) . \mathrm{PaO}_{2}$, arterial $\mathrm{O}_{2}$ tension; $\mathrm{PaCO}_{2}$, arterial $\mathrm{CO}_{2}$ tension.

$\dagger$ Indicates different from control at the $p<0.05$ level.

remains continuing uncertainty about how, when, and where fixed acids stimulate ventilation in developing and adult animals.

The time course and magnitude of the rise in blood lactate levels with this infusion were comparable to those observed with fetal asphyxia generated by partial uterine artery occlusion (6). 
It was in the 4 th and 5 th $h$ of that hypoxemic lactic acidosis that FBM returned to control. We did not measure lactate in our study of fetal anemia (5), but the fall in $\mathrm{pH}$ had a very similar time course as in the asphyxia (6) and the current lactic acid infusion. It should be remembered that, although we have simulated the peripheral blood lactic acidosis seen with fetal hypoxemia, the lactic acid concentration or $\mathrm{pH}$ at central chemoreceptive sites may not have simulated that which occurs with hypoxia. The fetal brain can generate lactate, and blood versus brain tissue concentration gradients may exist (19).

The increase in the incidence and depth of FBM in the 1 st $h$ of lactic acid infusion might be due to an effect of $\mathrm{pH}$ at peripheral arterial receptors. However, at least in adult dogs, this is a relatively weak respiratory stimulant (17). It also seems unlikely that this rapid but transient stimulation was the fixed acid penetrating to central chemoreceptors directly $(8,17)$. The slight stimulation may have been due to a transient increase in arterial $\mathrm{PCO}_{2}$ that results from the fixed acid being buffered by bicarbonate and stimulating central chemoreceptors (20). The $\mathrm{PaCO}_{2}$ measured in that $1 \mathrm{st} \mathrm{h}$ was somewhat elevated, an increase that approached statistical significance.

It is unclear why Johnston and Gluckman (21) found that lactic acid infusion in the $2 \mathrm{nd}$ and third $\mathrm{h}$ of infusion inhibited FBM in intact fetal sheep. Their results appear to be quite different from our current findings. Although they infused essentially similar amounts of lactic acid, the resultant arterial blood pH was slightly lower than that achieved in our current study. Perhaps the discrepancy was due to the type of lactic acid infused. We used the L optical isomer of lactic acid for our infusions; they did not specifically state which isomer they used. It is possible that different biologic transport properties of the optical isomers of lactic acid have caused different CNS conditions. We have previously noted that infusion of 0.2 normal $\mathrm{NaCl}$ appeared to inhibit FBM by an unknown mechanism (8). It may be that the volumes of distribution and osmotic impacts of different fixed acids can alter or mask the stimulatory effect of the hydrogen ion on central chemoreceptors. Their infusions resulted in a slightly greater fall in blood $\mathrm{pH}$ and perhaps brain lactic acid levels rose to some inhibitory range that we did not reach in our study.

Although we could not replicate the lactic acid inhibition of FBM in normoxic intact fetuses seen by Johnston and Gluckman (21), we observed some inhibitory element of lactic acid infusion in the hour after the induced hypoxemia. The mechanism, site, and biologic significance of this inhibition of FBM remain to be elucidated. The inhibition of FBM with lactic acid infusion reported by Johnston and Gluckman (21) became a stimulation in fetuses with discrete lesions of the lateral pons. These same lesions abolished the inhibition of FBM induced by acute hypoxemia. This particular area of the brainstem may be involved in sensing or processing the inhibitory neuromodulation of lactic acid.

Whatever the mechanism of the acute hypoxic depression, its adaptation, or the role of lactic acid in either phenomena in fetal sheep, it does not seem to act merely by modulating the incidence of the permissive LV EEG state, inasmuch as this was not altered by lactic acid infusion or by hypoxemia. The initial report by Boddy et al. (1) that hypoxemia reduced the incidence of the LV EEG state has not been replicated in more recent studies $(4,22)$.

Our finding of a slight but statistically significant decrease in breath amplitude during hypoxemia has not been found by other investigators $(3,4,22)$. Although it seems reasonable that the decrease in ventilatory drive that lowers the incidence of FBM with hypoxemia might also decrease the amplitude of breathing, it may be that subtle differences in analysis account for the difference between our findings and those of the others. We looked very carefully for low amplitude breathing and such breaths were included in our assessment of breathing depth. We cannot rule out that the trend (not statistically significant) toward a fall in $\mathrm{PCO}_{2}$ that we had in our study might have been mediated by the small decrease in breath amplitude we found with hypoxemia.

In summary, $6 \mathrm{~h}$ of lactic acid infusions did not alter the incidence of FBM for $4 \mathrm{~h}$ and did not significantly affect the inhibition of the incidence or depth of FBM during induced hypoxemia in the 5 th $\mathrm{h}$ of acidosis. Lactic acidosis did appear to prolong the hypoxemic respiratory inhibition into a subsequent normoxic recovery hour. A peripheral lactic acidemia does not appear to explain the adaptation of the respiratory inhibition seen with sustained hypoxemia. Rather, lactic acidemia may actually act as a respiratory inhibitor insofar as it marginally prolonged the inhibition of FBM into the time after the return to normoxemia.

Acknowledgments. The authors thank Sharon Knopp and Gail Willeke for their fine technical assistance and Keri Stepper for preparation of the manuscript.

\section{REFERENCES}

1. Boddy K, Dawes GS, Fisher R, Pinter S, Robinson JS 1974 Foetal respiratory movements, electrocortical and cardiovascular responses to hypoxaemia and hypercapnia in sheep. J Physiol (Lond) 243:599-618

2. Maloney JE, Adamson TM, Brodecky V, Dowling MH, Ritchie BC 1975 Modification of respiratory center output in the unanesthetized fetal sheep in utero. J Appl Physiol 39.552-558

3. Clewlow F, Dawes GS, Johnston BM, Walker DW 1983 Changes in breathing, electrocortical and muscle activity in unanaesthetized fetal lambs with age. J Physiol (Lond) 341:463-476

4. Sameshima H, Koos BJ 1986 Effects of moderate hypoxia on fetal electrocortical activity, eye movements, and breathing activity in sheep. J Dev Physiol $8: 411-419$

5. Bissonnette JM, Hohimer AR 1987 Acute anemic hypoxemia produces a transient depression in fetal respiratory activity. J Appl Physiol 63:19421946

6. Bissonnette JM, Hohimer AR, Willeke GB 1989 Effect of asphyxia on respiratory activity in fetal sheep. J Dev Physiol 12:157-161

7. Koos BJ, Kitanaka T, Matsuda K, Gilbert RD, Longo LD 1988 Fetal breathing adaptation to prolonged hypoxaemia in sheep. J Dev Physiol 10:161-166

8. Hohimer AR, Bissonnette JM 1981 Effect of metabolic acidosis of fetal breathing movements in utero. Respir Physiol 43:99-106

9. Gleed RD, Poore ER, Figueroa JP, Nathanielsz PW 1986 Modification of maternal and fetal oxygenation with the use of tracheal gas infusion. Am J Obstet Gynecol 155:429-435

10. Snedecor GW, Cochran WH 1980 Statistical Methods, 7th Ed. Iowa State University Press, Ames

11. Neubauer JA, Melton JE, Edelman NH 1990 Modulation of respiration during brain hypoxia. J Appl Physiol 68:441-451

12. Neubauer JA, Simone A, Edelman NH 1988 Role of brain lactic acidosis in hypoxic depression of respiration. J Appl Physiol 65:1324-1331

13. Davies DG 1978 Evidence for cerebral extracellular fluid $\left[\mathrm{H}^{+}\right]$as a stimulus during acclimatization to hypoxia. Respir Physiol 32:167-182

14. Dempsey JA, Forster HV 1982 Mediation of ventilatory adaptations. Physio Rev 62:262-347

15. Smith CA, Bisgard GE, Nielsen AM, Daristotle L, Kressin NA, Forster HV Dempsey JA 1986 Carotid bodies are required for ventilatory acclimatization to chronic hypoxia. J Appl Physiol 60:1003-1010

16. Molteni RA, Melmed MH, Sheldon RE, Jones MD, Meschia G 1980 Induction of fetal breathing by metabolic acidemia and its effect on blood flow to the respiratory muscles. Pediatr Res 136:609-620

17. Javaheri S, Herrera L, Kazemi H 1979 Ventilatory drive in acute metabolic acidosis. J Appl Physiol 45:913-918

18. Shirer HW, Erichsen DF, Orr JA 1988 Cardiorespiratory responses to HCL vs. lactic acid infusion. J Appl Physiol 65:534-540

19. Chao CR, Hohimer AR, Bissonnette JM 1989 Cerebral carbohydrate metab olism during severe ischemia in fetal sheep. J Cereb Blood Flow Metab 9:5357

20. Millhorn DE, Eldridge FL 1986 Role of ventrolateral medulla in regulation of respiratory and cardiovascular systems. J Appl Physiol 6I:1249-1263

21. Johnston BM, Gluckman PD 1989 Lateral pontine lesions affect central chemosensitivity in unanesthetized fetal lambs. J Appl Physiol 67:11131118

22. Adamson SL, Patrick JE, Challis JRG 1984 Effects of naloxone on breathing, electrocortical, heart rate, glucose and cortisol responses to hypoxia in the sheep fetus. J Dev Physiol 6:495-507 